\title{
The G-Asymptotic Tracking Property and G-Asymptotic Average Tracking Property in the Inverse Limit Spaces under Group Action
}

\author{
Zhanjiang Ji $\mathbb{D}^{1,2,3}$ \\ ${ }^{1}$ School of Data Science and Software Engineering, Wuzhou University, Wuzhou 54300, China \\ ${ }^{2}$ Guangxi Colleges and Universities Key Laboratory of Image Processing and Intelligent Information System, Wuzhou University, \\ Wuzhou 54300, China \\ ${ }^{3}$ Guangxi Colleges and Universities Key Laboratory of Professional Software Technology, Wuzhou University, \\ Wuzhou 54300, China
}

Correspondence should be addressed to Zhanjiang Ji; jizhanjiang1985@126.com

Received 28 January 2021; Accepted 22 April 2021; Published 12 May 2021

Academic Editor: Sundarapandian Vaidyanathan

Copyright $(2021$ Zhanjiang Ji. This is an open access article distributed under the Creative Commons Attribution License, which permits unrestricted use, distribution, and reproduction in any medium, provided the original work is properly cited.

Firstly, we introduce the definitions of $G$-asymptotic tracking property, $G$-asymptotic average tracking property, and $G$-quasiweak almost-periodic point. Secondly, we study their dynamical properties and characteristics. The results obtained improve the conclusions of asymptotic tracking property, asymptotic average tracking property, and quasi-weak almost-periodic point in the inverse limit space and provide the theoretical basis and scientific foundation for the application of tracking property in computational mathematics, biological mathematics, and computer science.

\section{Introduction}

Let $(X, d)$ be a metric space and let $f: X \longrightarrow X$ be a continuous map. The sequence $\left\{x_{i}\right\}_{i>0}$ is called $\delta$-pseudo orbit of $f$ if, for any $i \geq 0$, we have $d\left(f\left(x_{i}\right), x_{i+1}\right)<\delta$. The sequence $\left\{x_{i}\right\}_{i \geq 0}$ is said to be $\varepsilon$-shadowed by some point $y \in Y$ if, for any $i \geq 0$, we have $d\left(f(y), x_{i}\right)<\varepsilon$. The map $f$ is said to have the shadowing property if for each $\varepsilon>0$ there exists $\delta>0$ such that, for any $\delta$-pseudo orbit $\left\{x_{i}\right\}_{i \geq 0}$ of $f$, there exists a point $y \in X$ such that the sequence $\left\{x_{i}\right\}_{i \geq 0}$ is $\mathcal{E}$-shadowed by the point $y$ (see [1]).

The shadowing property plays an important role in ergodic theory and topological dynamical systems, which has attracted the attention of many scholars in recent years. The results are shown in literature [1-13]. In 1980, the concept of average tracking property was introduced by Blank [1] and it was proved that some perturbed hyperbolic systems have the average tracking property. In Wang and Zeng [2], the concept of $q$-average tracking property is given and the $q$-average tracking property means chain transitivity under some conditions. Fakhari and Ghane [3] introduced the concept of ergodic tracking property and discussed its dynamical properties. Liang and Li [4] discussed the relation between the shift mapping $\sigma$ and the self-mapping $f$ about the asymptotic tracking property in the inverse limit space. By the definitions of the $G$-tracking property of Ekta and Tarun [10], we introduce the concepts of $G$-asymptotic tracking property. By the definition of the asymptotic average tracking property of $\mathrm{Gu}$ [13], we give $G$-asymptotic average tracking property. The following conclusions are obtained: (1) The self-mapping $f$ has $G$-asymptotic tracking property if and only if the shift mapping $\sigma$ has $\bar{G}$-asymptotic tracking property. (2) The self-mapping $f$ has $G$-asymptotic average tracking property if and only if the shift mapping $\sigma$ has $\bar{G}$-asymptotic average tracking property. Thus, we generalize the conclusion of Liang and Li [4]. The quasi-weak almost-periodic point is an important concept in the dynamical system, which has also attracted the attention of many scholars. The relevant results are shown by $\mathrm{Ma}[14]$ and Zhou and $\mathrm{He}$ [15]. Ma [14] proved $Q W(\sigma)=\lim _{\leftarrow}(Q W(f), f)$. In this paper, we introduce the concept of $G$-quasi-weak almostperiodic point and study its topological structure in the inverse limit space under the action group. We obtain 
$Q W_{\bar{G}}(\sigma)=\lim _{\leftarrow}\left(Q W_{G}(f), f\right)$ and generalize the result of Ma [14].

\section{G-Asymptotic Tracking Property}

In this section, we will prove Theorem 1. For the convenience of the reader, we give the concept used in this section. Now we start with the following definitions.

Definition 1 (see [10]). Let $(X, d)$ be a metric space, let $G$ be a topological group, and let $\theta: G \times X \longrightarrow X$ be a continuous map. The triple $(X, G, \theta)$ is called a metric $G$-space if the following conditions are satisfied:

(1) $\theta(e, x)=x$ for all $x \in X$ and $e$ is the identity of $G$

(2) $\theta\left(g_{1}, \theta\left(g_{2}, x\right)\right)=\theta\left(g_{1} g_{2}, x\right)$ for all $x \in X$ and all $g_{1}, g_{2} \in G$

If $(X, d)$ is a compact metric space, then $(X, G, \theta)$ is also said to be a compact metric $G$-space. For the convenience of writing, $\theta(g, x)$ is usually abbreviated as $g x$.

Definition 2 (see [10]). Let $(X, d)$ be a metric $G$-space and let $f: X \longrightarrow X$ be a continuous map. The map $f$ is said to be an equivariant map if we have $f(p x)=p f(x)$ for all $x \in X$ and $p \in G$.

Definition 3 (see [16]). Let $(X, d)$ be a metric $G$-space and let $f: X \longrightarrow X$ be a continuous map. $\lim _{\leftarrow}(X, f)$ is said to be the inverse limit space if we write $\lim _{\leftarrow}(X, f)=\left\{\left(x_{0}, x_{1}, x_{2}, \ldots\right): f\left(x_{i+1}\right)=x_{i}, i \geq 0\right\}$.

$\lim _{\leftarrow}(X, f)$ is denoted by $X_{f}$ in this paper.

The metric $\bar{d}$ in $X_{f}$ is defined by $\bar{d}(\bar{x}, \bar{y})=\sum_{i=0}^{\infty}\left(d\left(x_{i}, y_{i}\right) / 2^{i}\right)$, where $\bar{x}=\left(x_{0}, x_{1}, x_{2} \ldots\right) \in X_{f}$ and $\bar{y}=\left(y_{0}, y_{1}, y_{2} \ldots\right) \in X_{f}$. The shift mapping $\sigma: X_{f} \longrightarrow X_{f}$ is defined by $\sigma(\bar{x})=\left(f\left(x_{0}\right), x_{0}, x_{1} \ldots\right)$. Thus, $\left(X_{f}, \vec{d}\right)$ is a compact metric space and the shift mapping $\sigma$ is a homeomorphism map.

Definition 4 (see $[16])$. Let $(X, d)$ be a metric $G$-space and let $f: X \longrightarrow X$ be an equivariant map. Write $\bar{G}=\{(g, g, g \ldots): g \in G\}$ and $G_{\infty}=\prod_{i=0}^{\infty} G_{i}$, where $G_{i}=G$. The map $\theta: \bar{G} \times X_{f} \longrightarrow X_{f}$ is defined by $\theta(\bar{g}, \bar{x})=\bar{g} \cdot \bar{x}=\left(g x_{0}, g x_{1}, g x_{2}, \ldots\right), \quad$ where $\bar{g}=(g, g, g \ldots) \in \bar{G}$ and $\bar{x}=\left(x_{0}, x_{1}, x_{2} \ldots\right) \in X_{f}$. Then $\left(X_{f}, \bar{G}, \theta\right)$ is a metric $\bar{G}$-space.

Let $\left(X_{f}, \bar{G}, \bar{d}, \sigma\right)$ and $(X, G, d, f)$ be shown as above. The space $\left(X_{f}, \bar{G}, \bar{d}, \sigma\right)$ is called the inverse limit space of $(X, G, d, f)$ under group action.

Definition 5 (see $[10])$. Let $(X, d)$ be a metric $G$-space and let $f: X \longrightarrow X$ be a continuous map. The sequence $\left\{x_{i}\right\}_{i \geq 0}$ is called be $(G, \delta)$-pseudo orbit of $f$ if for any $i \geq 0$ there exists $t_{i} \in G$ such that $d\left(t_{i} f\left(x_{i}\right), x_{i+1}\right)<\delta$.

Definition 6 (see [10]). Let $(X, d)$ be a metric $G$-space and let $f: X \longrightarrow X$ be a continuous map. The sequence $\left\{x_{i}\right\}_{i \geq 0}$ is said to be $(G, \delta)$-shadowed by some point $y \in Y$ if for any $i \geq 0$ there exists $t_{i} \in G$ such that $d\left(f(y), t_{i} x_{i}\right)<\delta$.
Definition 7 (see [10]). Let $(X, d)$ be a metric $G$-space and let $f: X \longrightarrow X$ be a continuous map. The map $f$ has $G$-tracking property if for each $\varepsilon>0$ there exists $\delta>0$ such that, for any $(G, \delta)$-pseudo orbit $\left\{x_{i}\right\}_{i \geq 0}$ of $f$, there exists a point $y \in Y$ such that the sequence $\left\{x_{i}\right\}_{i \geq 0}$ is $(G, \varepsilon)$-shadowed by the point $y$.

Remarks 1. By the definitions of the $G$-tracking property, we will give the concept of $G$-asymptotic tracking property.

Definition 8 Let $(X, d)$ be a metric $G$-space and let $f$ be a continuous map from $X$ to $X$. The map $f$ has $G$-asymptotic tracking property if for each $\varepsilon>0$ there exists $\delta>0$ such that, for any $(G, \delta)$-pseudo orbit $\left\{x_{i}\right\}_{i \geq 0}$ of $f$, there exists a point $y \in Y$ and $l \geq 0$ such that the sequence $\left\{x_{i}\right\}_{i=l}^{\infty}$ is $(G, \varepsilon)$-shadowed by the point $y$.

\section{Now, we start to prove Theorem 1 .}

Theorem 1. Let $\left(X_{f}, \bar{G}, \bar{d}, \sigma\right)$ be the inverse limit space of $(X, G, d, f)$ under group action. If the map $f: X \longrightarrow X$ is an equivalent surjection, we have that the self-mapping $f$ has the G-asymptotic tracking property if and only if the shift mapping $\sigma$ has the $\overline{\mathrm{G}}$-asymptotic tracking property.

Proof. $\Rightarrow$ Suppose that the map $f$ has the $G$-asymptotic tracking property. Since $X$ is compact, we write $M=\operatorname{diam}(X)$. For any $\varepsilon>0$, let $m>0$ satisfy $\left(M / 2^{m}\right)<(\varepsilon / 2)$. According to the fact that the map $f$ is uniformly continuous, for any $0 \leq i \leq m$, there exists $0<\delta_{1}<(\varepsilon / 4)$ such that $d(u, v)<\delta_{1}$ implies

$$
d\left(f^{i}(u), f^{i}(v)\right)<\frac{\varepsilon}{4} .
$$

By the definition of $G$-asymptotic tracking property, for $\delta_{1}>0$, there exists $0<\delta_{2}<\delta_{1}$ and $l_{1} \geq 0$ such that the map $f$ satisfies the condition of the $G$-asymptotic tracking property. Let $\left\{\bar{y}_{n}\right\}_{n=0}^{\infty}$ be $\left(\bar{G}, \delta_{2} / 2^{m}\right)$-pseudo orbit, where $\bar{y}_{n}=\left(y_{n}^{0}, y_{n}^{1}, y_{n}^{2} \ldots\right) \in X_{f}$. Then, for any $n \geq 0$, there exists $\bar{g}_{n}=\left(g_{n}, g_{n}, g_{n} \ldots\right) \in \bar{G}$ such that

$$
\bar{d}\left(\bar{g}_{n} \sigma\left(\bar{y}_{n}\right), \bar{y}_{n+1}\right)<\delta_{2} .
$$

Hence, we have that

$$
\frac{d\left(g_{n} f\left(y_{n}^{m}\right), y_{n+1}^{m}\right)}{2^{m}}<\frac{\delta_{2}}{2^{m}} .
$$

That is,

$$
d\left(g_{n} f\left(y_{n}^{m}\right), y_{n+1}^{m}\right)<\delta_{2} .
$$

So $\left\{y_{n}^{m}\right\}_{n=0}^{\infty}$ are $\left(G, \delta_{2}\right)$-pseudo orbit of the map $f$. Thus, for every $n \geq 0$, there exists $x \in X, t_{n} \in G$, and $l_{1} \geq 0$ such that

$$
d\left(f^{n}(x), t_{n} y_{n+l_{1}}^{m}\right)<\delta_{1} .
$$

According to (1) and the equivalent definition, for any $n \geq 0$ and $0 \leq i \leq m$, it follows that

$$
d\left(f^{n+i}(x), t_{n} y_{n+l_{1}}^{m-i}\right)<\frac{\varepsilon}{4} .
$$


Because of the surjectivity of the map $f$, we can choose $\bar{x}=\left(f^{m}(x), f^{m-1}(x), f^{m-2}(x), \ldots, x, \ldots\right) \in X_{f} \quad$ and $\bar{t}_{n}=\left(t_{n}, t_{n}, t_{n} \ldots\right) \in \bar{G}$. Then we have that

$$
\bar{d}\left(\sigma^{n}(\bar{x}), \bar{t}_{n} \bar{y}_{n+l_{1}}\right)<\sum_{i=0}^{m} \frac{\varepsilon}{2^{i+2}}+\sum_{i=m+1}^{\infty} \frac{M}{2^{i}}<\varepsilon .
$$

So, the map $f$ has the $G$-asymptotic tracking property.

$\Leftarrow$ Next we suppose that the shift mapping $\sigma$ has the $\bar{G}$-asymptotic tracking property. Let $n_{0}>0$. For any $\eta>0$, there exists $0<\delta_{3}<\eta$ and $l_{2} \geq 0$ such that, for any $\left(G, \delta_{3}\right)$-pseudo orbit $\left\{\bar{x}_{k}\right\}_{k=1}^{\infty}$ of the shift mapping $\sigma$, we have that $\left\{\bar{x}_{k}\right\}_{k=l 2}^{\infty}$ is $\left(\bar{G},\left(\eta / 2^{n_{0}}\right)\right)$-shadowed by the point $\bar{z}$ and $\left(M / 2^{n_{0}}\right)<\left(\delta_{3} / 2\right)$. Because the map $f$ is uniformly continuous, it follows that, for any $0 \leq i \leq n_{0}$, there exists $0<\delta_{4}<\left(\delta_{3} / 4\right)$ such that $d(u, v)<\delta_{4}$ implies

$$
d\left(f^{i}(u), f^{i}(v)\right)<\frac{\delta_{3}}{4}
$$

Now suppose that $\left\{x_{k}\right\}_{k=0}^{\infty}$ are $\left(G, \delta_{4}\right)$-pseudo orbit of the map $f$. Then, for any $k \geq 0$, there exists $s_{k} \in G$ such that

$$
d\left(s_{k} f\left(x_{k}\right), x_{k+1}\right)<\delta_{4} .
$$

According to (8) and the equivalent definition, for any $k \geq 0$ and $0 \leq i \leq n_{0}$, we have that

$$
d\left(s_{k} f^{i+1}\left(x_{k}\right), f^{i}\left(x_{k+1}\right)\right)<\frac{\delta_{3}}{4} .
$$

According to the surjectivity of the map $f$, we can choose $\bar{x}_{k}=\left(f^{n_{0}}\left(x_{k}\right), f^{n_{0}-1}\left(x_{k}\right), \ldots, f\left(x_{k}\right), x_{k}, \ldots\right) \in X_{f}$ and $\bar{s}_{k}=\left(s_{k}, s_{k}, s_{k}, \ldots\right) \in \bar{G}$. Combining $G(X)=X$ and (10), when $k \geq 0$, we have

$$
\bar{d}\left(\bar{s}_{k} \sigma\left(\bar{x}_{k}\right), \bar{x}_{k+1}\right)<\sum_{i=0}^{n_{0}} \frac{\delta_{3}}{2^{i+2}}+\sum_{i=n_{0}+1}^{\infty} \frac{M}{2^{n_{0}}}<\delta_{3} .
$$

So, $\left\{\bar{x}_{k}\right\}_{k=0}^{\infty}$ is $\left(\bar{G}, \delta_{3}\right)$-pseudo orbit of the shift mapping $\sigma$. By the definition of $\bar{G}$-asymptotic tracking property of the map $\sigma$, for any $k \geq 0$, there exists $\bar{z}=\left(z_{0}, z_{1}, z_{2} \ldots\right) \in X_{f}$, $l_{2} \geq 0$, and $\bar{p}_{k}=\left(p_{k}, p_{k}, p_{k} \ldots\right) \in \bar{G}$ such that

$$
\bar{d}\left(\sigma^{k}(\bar{z}), \bar{p}_{k} \bar{x}_{k+l_{2}}\right)<\frac{\eta}{2^{n_{0}}} .
$$

Thus, we have that

$$
\frac{d\left(f^{k}\left(z_{n_{0}}\right), p_{k} x_{k+l_{2}}\right)}{2^{n_{0}}}<\frac{\eta}{2^{n_{0}}} .
$$

Hence, we have that

$$
d\left(f^{k}\left(z_{n_{0}}\right), p_{k} x_{k+l_{2}}\right)<\eta .
$$

So the map $f$ has the $G$-asymptotic tracking property. Thus, we end the proof.

\section{G-Asymptotic Average Tracking Property}

Definition 9 (see [17]). Let $J \subset N$. If

$$
\lim _{n \longrightarrow \infty} \frac{\operatorname{Card}(J \cap\{0,1, \ldots, n-1\})}{n}=0,
$$

then the set $J$ is said to be a zero density set.

Definition 10 (see [13]). Let $(X, d)$ be a metric space and let $f: X \longrightarrow X$ be a continuous map. The sequence $\left\{x_{i}\right\}_{i \geq 0}$ in $X$ is called an asymptotic average pseudoorbit of the map $f$ if

$$
\lim _{n \longrightarrow \infty} \frac{1}{n} \sum_{i=0}^{i=n-1} d\left(f\left(x_{i}\right), x_{i+1}\right)=0 .
$$

Definition 11 (see [13]). Let $(X, d)$ be a metric space and let $f: X \longrightarrow X$ be a continuous map. The map $f$ is considered to have the asymptotic average tracking property if, for any asymptotic average pseudoorbit $\left\{x_{i}\right\}_{i \geq 0}$, there exists a point $z$ in $X$ such that

$$
\lim _{n \rightarrow \infty} \frac{1}{n} \sum_{i=0}^{i=n-1} d\left(f^{i}(z), x_{i}\right)=0 .
$$

Remarks 2. According to the definition of asymptotic average tracking property, we will give the concept of $G$-asymptotic average tracking property.

Definition 12. Let $(X, d)$ be a metric $G$-space and let $f: X \longrightarrow X$ be a continuous map. The sequence $\left\{x_{i}\right\}_{i \geq 0}$ in $X$ is called an $G$-asymptotic average pseudoorbit if there exists $t_{i} \in G$ such that

$$
\lim _{n \longrightarrow \infty} \frac{1}{n} \sum_{i=0}^{i=n-1} d\left(t_{i} f\left(x_{i}\right), x_{i+1}\right)=0 .
$$

Definition 13. Let $(X, d)$ be a metric $G$-space and let $f: X \longrightarrow X$ be a continuous map. The map $f$ is considered to have the $G$-asymptotic average tracking property if for any $G$-asymptotic average pseudo-orbit $\left\{x_{i}\right\}_{i \geq 0}$ there exists a point $z$ in $X$ and $t_{i} \in G$ such that

$$
\lim _{n \longrightarrow \infty} \frac{1}{n} \sum_{i=0}^{i=n-1} d\left(f^{i}(z), t_{i} x_{i}\right)=0 .
$$

Next, we give Lemma 1, which will be used in this section.

Lemma 1 (see [17]). Let $\left\{a_{i}\right\}_{i=0}^{\infty}$ be nonnegative real bounded sequence. Then the following conclusions are equivalent:

(1) $\lim _{n \longrightarrow \infty}(1 / n) \sum_{i=0}^{i=n-1} a_{i}=0$

(2) There exists a zero density set $J$ such that $\lim _{i \notin J, i \rightarrow \infty} a_{i}=0$

Now we will prove Theorem 2 by Lemma 1 .

Theorem 2. Let $\left(X_{f}, \bar{G}, \bar{d}, \sigma\right)$ be the inverse limit space of $(X, G, d, f)$ under group action. If the map $f: X \longrightarrow X$ is an equivalent surjection, we have that the self-mapping $f$ has the 
G-asymptotic average tracking property if and only if the shift mapping $\sigma$ has $\bar{G}$-asymptotic average tracking property.

Proof. $\Rightarrow$ Suppose that the map $f$ has the $G$-asymptotic average tracking property. Since $X$ is compact, we write $M=\operatorname{diam}(X)$. For any $\varepsilon>0$, let $m_{1}>0$ satisfy

$$
\frac{M}{2^{m_{1}}}<\frac{\varepsilon}{2} \text {. }
$$

According to the fact that the map $f$ is uniformly continuous, for any $0 \leq k \leq m_{1}$, there exists $0<\delta<(\varepsilon / 4)$ such that $d(u, v)<\delta$ implies

$$
d\left(f^{k}(u), f^{k}(v)\right)<\frac{\varepsilon}{4} .
$$

Let $\left\{\bar{y}_{i}\right\}_{i=0}^{\infty}$ be $\bar{G}$-asymptotic average pseudo-orbit, where $\bar{y}_{i}=\left(y_{i}^{0}, y_{i}^{1}, y_{i}^{2} \ldots\right) \in X_{f}$. Then there exists $\bar{g}_{i}=\left(g_{i}, g_{i}, g_{i} \ldots\right) \in \bar{G}$ such that

$$
\lim _{n \longrightarrow \infty} \frac{1}{n} \sum_{i=0}^{i=n-1} \bar{d}\left(\bar{g}_{i} \sigma\left(\bar{y}_{i}\right), \bar{y}_{i+1}\right)=0 .
$$

By Lemma 1, there exists a zero density set $J_{1}$ such that

$$
\lim _{i \notin J_{1}, i} \bar{d}\left(\bar{g}_{i} \sigma\left(\bar{y}_{i}\right), \bar{y}_{i+1}\right)=0 .
$$

Then, there exists $N_{1} \in N+$ such that when $i>N_{1}$ and $i \notin J_{1}$, we have that

$$
\bar{d}\left(\bar{g}_{i} \sigma\left(\bar{y}_{i}\right), \bar{y}_{i+1}\right)<\frac{\varepsilon}{2^{m_{1}}} .
$$

Thus, we have that

$$
\frac{d\left(g_{i} f\left(y_{i}^{m_{1}}\right), y_{i+1}^{m_{1}}\right)}{2^{m_{1}}}<\frac{\varepsilon}{2^{m_{1}}} .
$$

That is,

$$
d\left(g_{i} f\left(y_{i}^{m_{1}}\right), y_{i+1}^{m_{1}}\right)<\varepsilon .
$$

Hence, we have that

$$
\lim _{i \notin J_{1}, i} d\left(g_{i} f\left(y_{i}^{m_{1}}\right), y_{i+1}^{m_{1}}\right)=0 .
$$

According to Lemma 1, we have that

$$
\lim _{n \longrightarrow \infty} \frac{1}{n} \sum_{i=0}^{i=n-1} d\left(g_{i} f\left(y_{i}^{m_{1}}\right), y_{i+1}^{m_{1}}\right)=0 .
$$

Hence, the sequence $\left\{y_{i}^{m_{1}}\right\}_{i=0}^{\infty}$ is $G$-asymptotic average pseudoorbit of the map $f$. By the definition of $G$-asymptotic average tracking property of the map $f$, there exist $z \in X$ and $t_{i} \in G$ such that

$$
\lim _{n \longrightarrow \infty} \frac{1}{n} \sum_{i=0}^{i=n-1} d\left(f^{i}(z), t_{i} y_{i}^{m_{1}}\right)=0 .
$$

According to Lemma 1, there exists a zero density set $J_{2}$ such that

$$
\lim _{i \notin J_{1}, i \longrightarrow \infty} d\left(f^{i}(z), t_{i} y_{i}^{m_{1}}\right)=0
$$

Thus, there exists $N_{2} \in N+$ such that when $i>N_{2}$ and $i \notin J_{2}$, we have that

$$
d\left(f^{i}(z), t_{i} y_{i}^{m_{1}}\right)<\delta .
$$

According to the equivalent definition of the map $f$ and (21), for any $0 \leq k \leq m_{1}$, we have that

$$
d\left(f^{k+i}(z), t_{i} y_{i}^{m_{1}-k}\right)<\frac{\varepsilon}{4} .
$$

According to the surjectivity of the map $f$, we can choose $\bar{z}=\left(f^{m_{1}}(z), f^{m_{1}-1}(z), f^{m_{1}-2}(z), \ldots, z, \ldots\right) \in X_{f} \quad$ and $\bar{t}_{i}=\left(t_{i}, t_{i}, t_{i} \ldots\right) \in \bar{G}$. By (20) and (32), when $i>N_{2}$ and $i \notin J_{2}$, we have that

$$
\bar{d}\left(\sigma^{i}(\bar{z}), \bar{t}_{i} \bar{y}_{i}\right)<\varepsilon .
$$

So,

$$
\lim _{i \notin J_{2}, i \longrightarrow \infty} \bar{d}\left(\sigma^{i}(\bar{z}), \bar{t}_{i} \bar{y}_{i}\right)=0 .
$$

Combining with Lemma 1, we have that

$$
\lim _{n \longrightarrow \infty} \frac{1}{n} \sum_{i=0}^{i=n-1} \bar{d}\left(\sigma^{i}(\bar{z}), \bar{t}_{i} \bar{y}_{i}\right)=0 .
$$

So, the shift mapping $\sigma$ has the $\bar{G}$-asymptotic average tracking property.

$\Leftarrow$ Next we suppose that the shift mapping $\sigma$ has the $\bar{G}$-asymptotic average tracking property. For any $\eta>0$, let $m_{2}>0$ such that

$$
\frac{M}{2^{m_{2}}}<\frac{\eta}{2}
$$

Because the map $f$ is uniformly continuous, it follows that, for any $0 \leq k \leq m_{2}$, there exists $0<\delta^{\prime}<(\varepsilon / 4)$ such that $d(u, v)<\delta^{\prime}$ implies

$$
d\left(f^{k}(u), f^{k}(v)\right)<\frac{\eta}{4} .
$$

Now suppose that $\left\{x_{i}\right\}_{i=0}^{\infty}$ is $G$-asymptotic average pseudoorbit of the map $f$. Then there exists $p_{i} \in G$ such that

$$
\lim _{n \longrightarrow \infty} \frac{1}{n} \sum_{i=0}^{i=n-1} d\left(p_{i} f\left(x_{i}\right), x_{i+1}\right)=0 .
$$

By Lemma 1, there exists a zero density set $J_{3}$ such that

$$
\lim _{i \notin J_{3}, i \longrightarrow \infty} d\left(p_{i} f\left(x_{i}\right), x_{i+1}\right)=0 .
$$

Hence, there exists $N_{3} \in N_{+}$such that when $i>N_{3}$ and $i \notin J_{3}$, we have that

$$
d\left(p_{i} f\left(x_{i}\right), x_{i+1}\right)<\delta^{\prime} .
$$

According to the equivalent definition of the map $f$ and (37), for any $0 \leq k \leq m_{2}$, we have that

$$
d\left(p_{i} f^{k+1}\left(x_{i}\right), f^{k}\left(x_{i+1}\right)\right)<\frac{\eta}{4} .
$$

According to the surjectivity of the map $f$, we can choose $\bar{x}_{i}=\left(f^{m_{2}}\left(x_{i}\right), f^{m_{2}-1}\left(x_{i}\right), \ldots, f\left(x_{i}\right), x_{i}, \ldots\right) \in X_{f} \quad$ and 
$\bar{p}_{i}=\left(p_{i}, p_{i}, p_{i} \ldots\right) \in \bar{G}$, where $i \geq 0$. By (36) and (41), when $i>N_{3}$ and $i \notin J_{3}$, we have that

$$
\bar{d}\left(\bar{p}_{i} \sigma\left(\bar{x}_{i}\right), \bar{x}_{i+1}\right)<\sum_{i=0}^{m_{2}} \frac{\eta}{2^{i+2}}+\sum_{i=m_{2}+1}^{\infty} \frac{M}{2^{i}}<\eta .
$$

Hence, we have

$$
\lim _{i \notin J_{3}, i \longrightarrow \infty} \bar{d}\left(\bar{p}_{i} \sigma\left(\bar{x}_{i}\right), \bar{x}_{i+1}\right)=0 .
$$

Combining with Lemma 1, we can get that

$$
\lim _{n \longrightarrow \infty} \frac{1}{n} \sum_{i=0}^{i=n-1} \bar{d}\left(\bar{p}_{i} \sigma\left(\bar{x}_{i}\right), \bar{x}_{i+1}\right)=0 .
$$

So, $\left\{\bar{x}_{i}\right\}_{i=0}^{\infty}$ is $\bar{G}$-average pseudoorbit. By the definition of $\bar{G}$-asymptotic tracking property, there exist $\bar{y}=\left(y_{0}, y_{1}, y_{2}, \ldots\right) \in X_{f}$ and $\bar{s}_{i}=\left(s_{i}, s_{i}, s_{i}, \ldots\right) \in \bar{G}$ such that

$$
\lim _{n \rightarrow \infty} \frac{1}{n} \sum_{i=0}^{i=n-1} \bar{d}\left(\sigma^{i}(\bar{y}), \bar{s}_{i} \bar{x}_{i}\right)=0
$$

By Lemma 1, there exists a zero density set $J_{4}$ such that

$$
\lim _{i \notin J_{4}, i \longrightarrow \infty} \bar{d}\left(\sigma^{i}(\bar{y}), \bar{s}_{i} \bar{x}_{i}\right)=0 .
$$

Thus, we have that

$$
\lim _{i \notin J_{4}, i \longrightarrow \infty} d\left(f^{i}\left(y_{m_{2}}\right), s_{i} x_{i}\right)=0 .
$$

By Lemma 1, we have that

$$
\lim _{n \longrightarrow \infty} \frac{1}{n} \sum_{i=0}^{i=n-1} d\left(f^{i}\left(y_{m_{2}}\right), s_{i} x_{i}\right)=0 .
$$

So, the map $f$ has the $G$-asymptotic average tracking property. Thus, we end the proof.

\section{G-Quasi-Weak Almost-Periodic Point}

First, we give the definition that is used in this section. Second, we give the proof process of Theorem 3.

Definition 14 (see [18]). Let $(X, d)$ be a metric space and let $f: X \longrightarrow X$ be a continuous map. A point $x \in X$ is considered to be a quasi-weak almost-periodic point if, for any $\varepsilon>0$, there exists $N>0$ and nonnegative increasing integers sequence $\quad\left\{n_{i}\right\}_{i \geq 0}$ such that $\operatorname{Card}\left(\left\{r: f^{r}(x) \in B(x, \varepsilon), 0 \leq r<n_{i} N\right\}\right) \geq n_{i}$. The quasi-weak almost-periodic point set of the map $f$ is denoted by $Q W(f)$.

Remarks 3. According to the concept of quasi-weak almostperiodic point, we will give the concept of $G$-quasi-weak almost-periodic point.

Definition 15. Let $(X, d)$ be a metric $G$-space and let $f: X \longrightarrow X$ be a continuous map. A point $x \in X$ is considered to be a $G$-quasi-weak almost-periodic point if, for any $\varepsilon>0$, there exists $N>0$, nonnegative increasing integers sequence $\quad\left\{n_{i}\right\}_{i \geq 0}$, and $t_{i} \in G$ such that $\operatorname{Card}\left(\left\{r: t_{i} f^{r}(x) \in B(x, \varepsilon), 0 \leq r<n_{i} N\right\}\right) \geq n_{i}$. the $G$-quasiweak almost-periodic point set of the map $f$ is denoted by $Q W_{G}(f)$.

Now, we start to prove Theorem 3.

Theorem 3. Let $\left(X_{f}, \bar{G}, \bar{d}, \sigma\right)$ be the inverse limit space of $(X, G, d, f)$ under group action. If the map $f: X \longrightarrow X$ is an equivalent surjection, we have that $Q W_{\bar{G}}(\sigma)=\lim _{\leftarrow}$ $\left(Q W_{G}(f), f\right)$.

Proof. $\Rightarrow$ Suppose that $\bar{x}=\left(x_{0}, x_{1}, x_{2} \ldots\right) \in Q W_{\bar{G}}(\sigma)$. According to the definition of the $G$-quasi-weak almostperiodic point, for any $\varepsilon>0$, there exists $N>0$, nonnegative integer sequence $\left\{n_{i}\right\}_{i \geq 0}$, and $\bar{g}_{i}=\left(g_{0}, g_{1}, g_{2} \ldots\right) \in \bar{G}$ such that

$$
\operatorname{Card}\left(\left\{r: \bar{g}_{i} \sigma^{r}(\bar{x}) \in B\left(\bar{x}, \frac{\varepsilon}{2^{i}}\right), 0 \leq r<n_{i} N\right\}\right) \geq n_{i} .
$$

Write

$$
\begin{aligned}
& A_{n_{i}}=\left\{r: \bar{g}_{i} \sigma^{r}(\bar{x}) \in B\left(\bar{x}, \frac{\varepsilon}{2^{i}}\right), 0 \leq r<n_{i} N\right\}, \\
& B_{n_{i}}=\left\{r: g_{i} f^{r}\left(x_{i}\right) \in B\left(x_{i}, \varepsilon\right), 0 \leq r<n_{i} N\right\} .
\end{aligned}
$$

Now, suppose that $r \in A_{n_{i}}$. Then we have that

$$
\bar{d}\left(\bar{g}_{i} \sigma^{r}(\bar{x}), \bar{x}\right)<\frac{\varepsilon}{2^{i}} .
$$

Hence, we have that

$$
d\left(g_{i} f^{r}\left(x_{i}\right), x_{i}\right)<\varepsilon
$$

Thus, we can obtain $r \in B_{n_{i}}$. So, we get that

$$
\operatorname{Card}\left(\left\{r: g_{i} f^{r}\left(x_{i}\right) \in B\left(x_{i}, \varepsilon\right), 0 \leq r<n_{i} N\right\}\right) \geq n_{i} \text {. }
$$

So, $Q W_{\bar{G}}(\sigma) \subset \lim _{\leftarrow}\left(Q W_{G}(f), f\right)$.

$\stackrel{\text { Suppose }}{\Leftarrow} \quad$ that $\bar{y}=\left(y_{0}, y_{1}, y_{2}, \ldots\right) \in \lim _{\leftarrow}\left(Q W_{G}(f), f\right)$. Then, for any $i \geq 0$ , we have $y_{i} \in Q W_{G}(f)$. Since $X$ is compact, we write $M=\operatorname{diam}(X)$. For any $\eta>0$, let $m>0$ satisfy

$$
\frac{M}{2^{m}}<\frac{\eta}{2}
$$

According to $y_{i} \in Q W_{G}(f)$, there exists $N>0$, nonnegative integer sequence $\left\{m_{i}\right\}_{i \geq 0}$, and $t_{i} \in G$ such that

$\operatorname{Card}\left(\left\{r: t_{i} f^{r}\left(y_{i}\right) \in B\left(y_{i}, \eta\right), 0 \leq r<m_{i} N\right\}\right) \geq m_{i}$.

Let $\bar{t}=\left(t_{0}, t_{1}, t_{2}, \ldots\right)$. Write

$$
\begin{aligned}
& C_{m_{i}}=\left\{r: t_{i} f^{r}\left(y_{i}\right) \in B\left(y_{i}, \frac{\eta}{4}\right), 0 \leq r<m_{i} N\right\}, \\
& D_{m_{i}}=\left\{r: \bar{t} \sigma^{r}(\bar{y}) \in B(\bar{y}, \eta), 0 \leq r<m_{i} N\right\} .
\end{aligned}
$$

Suppose that $r \in C_{m_{i}}$. Then we have that 


$$
d\left(t_{i} f^{r}\left(y_{i}\right), y_{i}\right)<\frac{\eta}{4}
$$

Thus, we have that

$$
\begin{aligned}
\bar{d}\left(\bar{t} \sigma^{r}(\bar{y}), \bar{y}\right) & =\sum_{i=0}^{m} \frac{d\left(t_{i} f^{r}\left(y_{i}\right), y_{i}\right)}{2^{i}}+\sum_{i=m+1}^{\infty} \frac{d\left(t_{i} f^{r}\left(y_{i}\right), y_{i}\right)}{2^{i}} \\
& <\sum_{i=0}^{m} \frac{1}{2^{i}} \cdot \frac{\eta}{4}+\sum_{i=m+1}^{\infty} \frac{M}{2^{i}} \\
& <\frac{\eta}{2}+\frac{\eta}{2}=\eta .
\end{aligned}
$$

Thus, we can obtain $r \in D_{m_{i}}$. So we get that

$$
\operatorname{Card}\left(\left\{r: \bar{t} \sigma^{r}(\bar{y}) \in B(\bar{y}, \eta), 0 \leq r<m_{i} N\right\}\right) \geq m_{i} \text {. }
$$

Hence, $\quad \bar{y} \in Q W_{\bar{G}}(\sigma)$. Then $\quad \lim \longrightarrow\left(Q W_{G}\right.$ $(f), f) \subset Q W_{\bar{G}}(\sigma)$. This completes the proof.

\section{Conclusions}

In this paper, we study dynamical properties and characteristics of $G$-asymptotic tracking property, $G$-asymptotic average tracking property, and $G$-quasi-weak almost-periodic point. The results obtained can generalize the conclusions of asymptotic tracking property, asymptotic average tracking property, and quasi-weak almost-periodic point in the inverse limit space. Most importantly, the paper provides the theoretical basis and scientific foundation for the application of tracking property in computational mathematics, biological mathematics, nature, and society.

\section{Data Availability}

No data were used to support this study.

\section{Conflicts of Interest}

The authors declare that they have no conflicts of interest.

\section{Acknowledgments}

This work was partially supported by the NSF of Guangxi Province (2020JJA110021) and construction project of Wuzhou University of China (2020B007).

\section{References}

[1] M. L. Blank, "Metric properties of $\varepsilon$-trajectories of dynamical systems with stochastic behaviour," Ergodic Theory and Dynamical Systems, vol. 8, no. 3, pp. 365-378, 1988.

[2] H. Y. Wang and P. Zeng, "Partial shadowing of averagepseudo-orbits," Scientia Sinica (Mathematica), vol. 46, no. 4, pp. 781-792, 2016.

[3] A. Fakhari and F. H. Ghane, "On shadowing: ordinary and ergodic," Journal of Mathematical Analysis and Applications, vol. 364, no. 1, pp. 151-155, 2010.
[4] C. Liang and S. H. Li, "Shadowing property for inverse limit spaces," Proceedings of the American Mathematical Society, vol. 115, no. 2, pp. 573-580, 1992.

[5] X. Wu, "Some remarks on $d$-shadowing property," Scientia Sinica Mathematica, vol. 45, no. 3, pp. 273-286, 2015.

[6] D. D. Ahmadi and M. Hosseini, "Sub-shadowings," Nonlinear Analysis, vol. 72, pp. 3759-3766, 2010.

[7] J.-J. Park and Y. Zhang, "Average shadowing properties on compact metric spaces," Communications of the Korean Mathematical Society, vol. 21, no. 2, pp. 355-361, 2006.

[8] P. Oprocha, D. A. Dastjerdi, and M. Hosseini, "On partial shadowing of complete pseudo-orbits," Journal of Mathematical Analysis and Applications, vol. 404, no. 1, pp. 47-56, 2013.

[9] D. Kwietniak and P. Oprocha, "A note on the average shadowing property for expansive maps," Topology and Its Applications, vol. 159, no. 1, pp. 19-27, 2012.

[10] S. Ekta and D. Tarun, "Consequences of shadowing property of G-spaces," Math Analysis, vol. 7, no. 12, pp. 579-588, 2013.

[11] Y. Niu, "The average-shadowing property and strong ergodicity," Journal of Mathematical Analysis and Applications, vol. 376, no. 2, pp. 528-534, 2011.

[12] M. Kulczycki, D. Kwietniak, and P. Oprocha, "On almost specification and average shadowing properties," Fundamenta Mathematicae, vol. 224, no. 3, pp. 241-278, 2014.

[13] R. Gu, "The asymptotic average shadowing property and transitivity," Nonlinear Analysis: Theory, Methods \& Applications, vol. 67, no. 6, pp. 1680-1689, 2007.

[14] D. K. Ma, "Some properties about the shift maps on the inverse limit spaces," Acta Scientiarum Naturalium Universitatis Sunyatseni, vol. 37, no. 6, pp. 10-14, 1998.

[15] Z. L. Zhou and W. H. He, "Hierarchy of track structure and topological semi-conjugate," Scientia Sinica (Mathematica), vol. 5, pp. 457-464, 1995.

[16] Z. J. Ji, Dynamical Property of Product Space and the Inverse Limit Space of a Topological Group Action, Guangxi University, Nanning, China, 2014.

[17] R. Gu, Y. Sheng, and Z. Xia, "The average-shadowing property and transitivity for continuous flows," Chaos, Solitons \& Fractals, vol. 23, no. 3, pp. 989-995, 2005.

[18] Z. J. Ji, "The research of quasi-weak almost periodic point and sequence shadowing property under strongly uniform convergence," Journal of Southwest China Normal University (Natural Science Edition), vol. 44, no. 12, pp. 40-44, 2019. 